\title{
On the use of the Obara-Saika recurrence relations for the calculation of structure factors in quantum crystallography
}

\section{Alessandro Genoni}

Acta Cryst. (2020). A76, 172-179

\section{IUCr Journals CRYSTALLOGRAPHY JOURNALS ONLINE}

Copyright (C) International Union of Crystallography

Author(s) of this article may load this reprint on their own web site or institutional repository provided that this cover page is retained. Republication of this article or its storage in electronic databases other than as specified above is not permitted without prior permission in writing from the IUCr.

For further information see https://journals.iucr.org/services/authorrights.html 


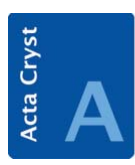

FOUNDATIONS

ADVANCES

ISSN 2053-2733

Received 7 October 2019

Accepted 14 January 2020

Edited by P. M. Dominiak, University of Warsaw, Poland

Keywords: quantum crystallography; structure factors; Fourier transform integrals; Obara-Saika recurrence relations.

Supporting information: this article has supporting information at journals.iucr.org/a

\section{On the use of the Obara-Saika recurrence relations for the calculation of structure factors in quantum crystallography}

\author{
Alessandro Genoni* \\ Université de Lorraine and CNRS, Laboratoire de Physique et Chimie Théoriques, 1 Boulevard Arago, F-57078 Metz, \\ France. *Correspondence e-mail: alessandro.genoni@univ-lorraine.fr
}

Modern methods of quantum crystallography are techniques firmly rooted in quantum chemistry and, as in many quantum chemical strategies, electron densities are expressed as two-centre expansions that involve basis functions centred on atomic nuclei. Therefore, the computation of the necessary structure factors requires the evaluation of Fourier transform integrals of basis function products. Since these functions are usually Cartesian Gaussians, in this communication it is shown that the Fourier integrals can be efficiently calculated by exploiting an extension of the Obara-Saika recurrence formulas, which are successfully used by quantum chemists in the computation of molecular integrals. Implementation and future perspectives of the technique are also discussed.

\section{Introduction}

Quantum crystallography $(\mathrm{QCr})$ is a branch of crystallography characterized by a strict interplay between quantum mechanical and crystallographic information (Genoni, Bučinský et al., 2018; Novara et al., 2018; Grabowsky et al., 2017; Massa \& Matta, 2018; Tsirelson, 2018). This interplay was already clear in the traditional and well known multipole model methods for the determination of experimental charge densities (Hirshfeld, 1971; Stewart, 1976; Kurki-Suonio, 1977; Hansen \& Coppens, 1978; Tsirelson \& Ozerov, 1996; Coppens, 1997) and in the pioneering techniques for the determination of diagonal and off-diagonal elements of one-particle density matrices (Clinton \& Massa, 1972; Clinton et al., 1973; Schmider et al., 1992; Gillet et al., 2001; Gillet, 2007). However, it became even more evident in the modern methods of QCr (Grabowsky et al., 2017), (i) where the results obtained from quantum chemistry calculations are directly exploited in structural refinements to complete the information content of crystallographic measurements [e.g. in the Hirshfeld atom refinement (HAR) technique (Jayatilaka \& Dittrich, 2008; Capelli et al., 2014; Woińska et al., 2014; Wall, 2016; Woińska et al., 2016; Fugel et al., 2018, 2019; Malaspina et al., 2019)] or (ii) where the experimental crystallographic data are directly used in quantum mechanical computations to enhance the information content of the wavefunction [e.g. in the X-ray constrained wavefunction (XCW) fitting approach (Jayatilaka, 1998; Jayatilaka \& Grimwood, 2001; Grimwood \& Jayatilaka, 2001; Bytheway et al., 2002; Bytheway, Grimwood, Figgis et al., 2002; Grimwood et al., 2003; Hudák et al., 2010; Jayatilaka, 2012)]. Since these new strategies are firmly rooted in quantum chemistry, the electron-density distributions are generally written as two-centre expansions of Cartesian Gaussian-type functions (GTFs) centred on atomic nuclei. This leads to the 
fact that, for the computation of the needed structure factors, it is necessary to evaluate Fourier integrals of products of Cartesian Gaussians.

Over the years several research groups have faced this problem, from the initial efforts by McWeeny (1953), Chandler \& Spackman (1978) and Barua \& Weyrich (1986), to the more recent work of the Kirrander group in the context of the $a b$ initio X-ray diffraction (AIXRD) method (Northey et al., 2014; Moreno Carrascosa et al., 2019; Northey \& Kirrander, 2019). However, so far in this context the most efficient technique remains the one proposed by Jayatilaka (1994), who extended the McMurchie-Davidson (MD) recurrence relations used in quantum chemistry to evaluate molecular integrals (McMurchie \& Davidson, 1978). This is actually the strategy used for the computation of structure factors in all the XCW methods implemented in the quantum crystallographic software Tonto (Jayatilaka \& Grimwood, 2003).

The MD approach is only one of the many efficient strategies proposed and used by quantum chemists to compute molecular integrals efficiently. Many excellent reviews are currently available on the subject (Clementi \& Corongiu, 1989; Gill, 1994; Helgaker et al., 2000; Reine et al., 2012). Among these techniques, one of the most popular and efficient is the strategy based on the recurrence relations introduced by Obara \& Saika (1986), which were also discussed in the context of the Fourier transform operation in a follow-up paper (Obara \& Saika, 1988). The Obara-Saika (OS) approach has actually been used very recently to compute structure factors corresponding to quantum chemistry-like electron densities: in XCW methods (Genoni, 2013a,b; Dos Santos et al., 2014; Genoni \& Meyer, 2016; Genoni, 2017; Casati et al., 2017) based on the concept of extremely localized molecular orbitals (Stoll et al., 1980; Meyer, Guillot, RuizLopez \& Genoni, 2016a; Meyer, Guillot, Ruiz-Lopez, Jelsch \& Genoni, 2016b; Meyer \& Genoni, 2018), in the novel X-ray constrained spin-coupled technique (Genoni, Franchini et al., 2018; Genoni et al., 2019), in charge-density investigations of molecular magnets (Thomsen et al., 2019; Gao et al., 2019) and in the study of the capability of the XCW strategy in capturing electron correlation effects on electron density (Genoni et al., 2017).

However, in all the cases mentioned above, the use of OS recurrence relations for the calculation of structure factors has barely been mentioned, and never discussed in detail. Therefore, the goal of this article is to fill this gap, by showing the theoretical details and the derivation of the extended OS formulas for theoretical structure factors, their implementation and, where possible, their comparison with other existing approaches.

\section{Theory}

\subsection{Structure factors and electron-density expressions}

As is well known (Tsirelson \& Ozerov, 1996; Coppens, 1997; Schwarzenbach, 1996; Giacovazzo, 2002), structure factors are
Fourier transforms of the space- and time-averaged unit-cell electron density $\bar{\rho}_{\text {cell }}(\mathbf{r})$,

$$
F_{\mathbf{H}}=\int \mathrm{d} \mathbf{r} \bar{\rho}_{\text {cell }}(\mathbf{r}) \exp (i 2 \pi \mathbf{H} \cdot \mathbf{r}),
$$

with $\mathbf{H}$ the product of the reciprocal-lattice matrix and the vector representing the triad of Miller indices associated with the reflection under consideration. If one exploits the unit-cell symmetry operations $\left\{\mathbf{R}_{j}, \mathbf{t}_{j}\right\}_{j=1}^{N_{m}}$ (with $\mathbf{R}_{j}$ and $\mathbf{t}_{j}$ as a rotation matrix and a translation vector, respectively), $\bar{\rho}_{\text {cell }}(\mathbf{r})$ can actually be written in terms of the space- and time-averaged electron density $\bar{\rho}_{0}(\mathbf{r})$ of the symmetry-independent fragment in the unit cell. Equation (1) thus becomes

$$
F_{\mathbf{H}}=\sum_{j=1}^{N_{m}} \exp \left(i 2 \pi \mathbf{t}_{j} \cdot \mathbf{H}\right) \int \mathrm{d} \mathbf{r} \bar{\rho}_{0}(\mathbf{r}) \exp \left[i 2 \pi\left(\mathbf{R}_{j}^{\mathrm{T}} \mathbf{H}\right) \cdot \mathbf{r}\right] .
$$

The averaged density $\bar{\rho}_{0}(\mathbf{r})$ can be expressed as the convolution of the static electron density $\rho_{0}(\mathbf{r})$ with the probability distribution function $P\left(\mathbf{u}_{1}, \mathbf{u}_{2}, \ldots, \mathbf{u}_{N}\right)$ for the nuclear positions, namely

$$
\bar{\rho}_{0}(\mathbf{r})=\rho_{0}(\mathbf{r}) \star P\left(\mathbf{u}_{1}, \mathbf{u}_{2}, \ldots, \mathbf{u}_{N}\right) .
$$

In the most recent methods of quantum crystallography (i.e. HAR and XCW techniques), the static electron density is written as a quantum chemistry-like electron distribution and, in the most general way (which is valid for both singledeterminant and multi-determinant wavefunction-based approaches), it can be expressed in terms of natural orbitals,

$$
\rho_{0}(\mathbf{r})=\sum_{i=1}^{M} n_{i} \phi_{i}^{*}(\mathbf{r}) \phi_{i}(\mathbf{r})
$$

with $\phi_{i}$ the generic $i$ th natural orbital associated with the obtained wavefunction, $\phi_{i}^{*}$ the complex conjugate of $\phi_{i}, n_{i}$ the occupation number for the $i$ th natural orbital $\left(0 \leq n_{i} \leq 2\right)$ and $M$ the total number of basis functions used in the calculation. It is worth pointing out that, in the case of a single-determinant wavefunction, the natural orbitals correspond to the more usual molecular orbitals (MOs) and the occupation numbers are equal to 2 for the occupied MOs and 0 for the virtual ones.

In quantum chemistry, molecular orbitals are generally expanded in terms of basis functions centred on nuclei:

$$
\phi_{i}(\mathbf{r})=\sum_{\alpha=1}^{M} C_{\alpha i} \chi_{\alpha}(\mathbf{r}-\mathbf{A}),
$$

where $\mathbf{A}=\left(A_{x}, A_{y}, A_{z}\right)$ is the position vector of the nucleus $A$ on which the basis function $\chi_{\alpha}$ is centred and the quantities $C_{\alpha i}$ are the molecular orbital expansion coefficients. Therefore, equation (4) can be rewritten as

$$
\rho_{0}(\mathbf{r})=\sum_{\alpha=1}^{M} \sum_{\beta=1}^{M} \chi_{\alpha}(\mathbf{r}-\mathbf{A}) D_{\alpha \beta}^{0} \chi_{\beta}(\mathbf{r}-\mathbf{B}),
$$

with the one-electron density matrix $\mathbf{D}^{0}$ defined as 


$$
D_{\alpha \beta}^{0}=\sum_{i=1}^{M} n_{i} C_{\alpha i}^{*} C_{\beta i} .
$$

It is clear that the expression of $\rho_{0}(\mathbf{r})$ given by equation (6) is an expansion involving basis functions $\chi_{\alpha}$ and $\chi_{\beta}$, which can possibly be centred on two different nuclei. If we use this expression for the static electron density in equation (3) and, consequently, in equation (2), and if we exploit the convolution theorem, the structure-factor expression becomes

$$
F_{\mathbf{H}}=\sum_{\alpha=1}^{M} \sum_{\beta=1}^{M} D_{\alpha \beta}^{0} I_{\beta \alpha}^{\mathbf{H}}=\operatorname{Tr}\left[\mathbf{D}^{0} \mathbf{I}^{\mathbf{H}}\right],
$$

where $\mathbf{I}^{\mathbf{H}}$ is the matrix of the averaged Fourier integrals of the basis function products, namely the matrix having elements

$$
\begin{aligned}
I_{\alpha \beta}^{\mathbf{H}}= & \sum_{j=1}^{N_{m}} \exp \left(i 2 \pi \mathbf{t}_{j} \cdot \mathbf{H}\right) F[P(\mathbf{A}, \mathbf{B})] \\
& \times \int \mathrm{d} \mathbf{r} \chi_{\alpha}(\mathbf{r}-\mathbf{A}) \chi_{\beta}(\mathbf{r}-\mathbf{B}) \exp \left[i 2 \pi\left(\mathbf{R}_{j}^{\mathrm{T}} \mathbf{H}\right) \cdot \mathbf{r}\right],
\end{aligned}
$$

with $F[P(\mathbf{A}, \mathbf{B})]$ as the Fourier transform of the probability distribution function for the nuclei $A$ and $B$ on which the basis functions $\chi_{\alpha}$ and $\chi_{\beta}$ are centred, respectively. Since $P(\mathbf{A}, \mathbf{B})$ is not available, the factor $F[P(\mathbf{A}, \mathbf{B})]$ is usually approximated through methods that use parameters related to one-atom probability distribution functions, namely, the usual anisotropic displacement parameters (Stewart, 1969; Coppens et al., 1971; Tanaka, 1988; Michael \& Koritsanszky, 2015).

The other important factor in equation (9) is the Fourier integral of the product of basis functions. By setting $\mathbf{k}=$ $2 \pi\left(\mathbf{R}_{j}^{\mathrm{T}} \mathbf{H}\right)$, that factor can be rewritten as

$$
I=\int \mathrm{d} \mathbf{r} \chi_{\alpha}\left(\mathbf{r}-\mathbf{A} ; L_{\alpha}\right) \chi_{\beta}\left(\mathbf{r}-\mathbf{B} ; L_{\beta}\right) \exp (i \mathbf{k} \cdot \mathbf{r}),
$$

where, for the two basis functions, we have also explicitly indicated their total angular momenta $L_{\alpha}$ and $L_{\beta}$.

\subsection{Recurrence relations for integrals}

In quantum chemical calculations, the adopted basis functions are generally contracted Cartesian Gaussian-type functions (GTFs). In other words, they are linear combinations of primitive Cartesian GTFs (Boys, 1950):

$$
\chi_{\alpha}(\mathbf{r}-\mathbf{A})=\sum_{i=1}^{K_{\alpha}} N_{i \alpha} C_{i \alpha} g_{i \alpha}\left(\mathbf{r}-\mathbf{A} ; L_{\alpha}\right)
$$

and

$$
\chi_{\beta}(\mathbf{r}-\mathbf{B})=\sum_{j=1}^{K_{\beta}} N_{j \beta} C_{j \beta} g_{j \beta}\left(\mathbf{r}-\mathbf{B} ; L_{\beta}\right),
$$

where $K_{\alpha}$ and $K_{\beta}$ are the degrees of contraction, $N_{i \alpha}$ and $N_{j \beta}$ are normalization constants, $C_{i \alpha}$ and $C_{j \beta}$ are the contraction coefficients for the primitive functions, and $g_{i \alpha}\left(\mathbf{r}-\mathbf{A} ; L_{\alpha}\right)$ and $g_{j \beta}\left(\mathbf{r}-\mathbf{B} ; L_{\beta}\right)$ are un-normalized primitive Cartesian Gaus- sian-type functions centred on the nuclear positions $\mathbf{A}$ and $\mathbf{B}$ and of total angular momenta $L_{\alpha}$ and $L_{\beta}$, respectively.

Substituting (11) and (12) into (10), the integral $I$ becomes

$$
\begin{aligned}
I= & \sum_{i=1}^{K_{\alpha}} \sum_{j=1}^{K_{\beta}} N_{i \alpha} N_{j \beta} C_{i \alpha} C_{j \beta} \\
& \times \int \mathrm{d} \mathbf{r} g_{i \alpha}\left(\mathbf{r}-\mathbf{A} ; L_{\alpha}\right) g_{j \beta}\left(\mathbf{r}-\mathbf{B} ; L_{\beta}\right) \exp (i \mathbf{k} \cdot \mathbf{r}) \\
= & \sum_{i=1}^{K_{\alpha}} \sum_{j=1}^{K_{\beta}} N_{i \alpha} N_{j \beta} C_{i \alpha} C_{j \beta} I_{i \alpha}^{j \beta} .
\end{aligned}
$$

The un-normalized primitive Cartesian GTFs are usually written as

$$
\begin{aligned}
g_{i \alpha}\left(\mathbf{r}-\mathbf{A} ; L_{\alpha}\right) & \\
= & \left(x-A_{x}\right)^{a_{x}}\left(y-A_{y}\right)^{a_{y}}\left(z-A_{z}\right)^{a_{z}} \\
& \quad \times \exp \left\{-\alpha_{i}\left[\left(x-A_{x}\right)^{2}+\left(y-A_{y}\right)^{2}+\left(z-A_{z}\right)^{2}\right]\right\}
\end{aligned}
$$

and

$$
\begin{aligned}
g_{j \beta}\left(\mathbf{r}-\mathbf{B} ; L_{\beta}\right) & \\
= & \left(x-B_{x}\right)^{b_{x}}\left(y-B_{y}\right)^{b_{y}}\left(z-B_{z}\right)^{b_{z}} \\
& \quad \times \exp \left\{-\beta_{j}\left[\left(x-B_{x}\right)^{2}+\left(y-B_{y}\right)^{2}+\left(z-B_{z}\right)^{2}\right]\right\} .
\end{aligned}
$$

$g_{i \alpha}$ is an un-normalized Cartesian GTF centred on $\mathbf{A}=$ $\left(A_{x}, A_{y}, A_{z}\right)$, with angular momentum indices $\mathbf{a}=\left(a_{x}, a_{y}, a_{z}\right)$ and total angular momentum $L_{\alpha}=a_{x}+a_{y}+a_{z}$, while $g_{j \beta}$ is an un-normalized Cartesian GTF centred on $\mathbf{B}=\left(B_{x}, B_{y}, B_{z}\right)$, with angular momentum indices $\mathbf{b}=\left(b_{x}, b_{y}, b_{z}\right)$ and total angular momentum $L_{\beta}=b_{x}+b_{y}+b_{z}$.

Making use of the Gaussian product rule (Gill, 1994; Helgaker et al., 2000), we can set

$$
E_{i \alpha}^{j \beta}=\exp \left[-\frac{\alpha_{i} \beta_{j}}{\alpha_{i}+\beta_{j}}(\mathbf{A}-\mathbf{B})^{2}\right]
$$

and

$$
\mathbf{P}=\frac{\alpha_{i} \mathbf{A}+\beta_{j} \mathbf{B}}{\alpha_{i}+\beta_{j}} .
$$

Therefore, if we introduce equations (14) and (15) into (13), the integral $I_{i \alpha}^{j \beta}$ becomes

$$
\begin{aligned}
I_{i \alpha}^{j \beta}= & E_{i \alpha}^{j \beta} \int \mathrm{d} x \exp \left(i k_{x} x\right)\left(x-A_{x}\right)^{a_{x}}\left(x-B_{x}\right)^{b_{x}} \\
& \times \exp \left[-\left(\alpha_{i}+\beta_{j}\right)\left(x-P_{x}\right)^{2}\right] \\
& \times \int \mathrm{d} y \exp \left(i k_{y} y\right)\left(y-A_{y}\right)^{a_{y}}\left(y-B_{y}\right)^{b_{y}} \\
& \times \exp \left[-\left(\alpha_{i}+\beta_{j}\right)\left(y-P_{y}\right)^{2}\right] \\
& \times \int \mathrm{d} z \exp \left(i k_{z} z\right)\left(z-A_{z}\right)^{a_{z}}\left(z-B_{z}\right)^{b_{z}} \\
& \times \exp \left[-\left(\alpha_{i}+\beta_{j}\right)\left(z-P_{z}\right)^{2}\right],
\end{aligned}
$$

which, in short notation, is equivalent to 


$$
\begin{aligned}
I_{i \alpha}^{j \beta}(\mathbf{a} \mid \mathbf{b}) & =I_{i \alpha}^{j \beta}\left(a_{x}, a_{y}, a_{z} \mid b_{x}, b_{y}, b_{z}\right) \\
& =E_{i \alpha}^{j \beta} I_{x}\left(a_{x} \mid b_{x}\right) I_{y}\left(a_{y} \mid b_{y}\right) I_{z}\left(a_{z} \mid b_{z}\right) .
\end{aligned}
$$

Now, let us focus only on the factor $I_{x}\left(a_{x} \mid b_{x}\right)$ :

$$
\begin{aligned}
I_{x}\left(a_{x} \mid b_{x}\right)= & \int \mathrm{d} x \exp \left(i k_{x} x\right)\left(x-A_{x}\right)^{a_{x}}\left(x-B_{x}\right)^{b_{x}} \\
& \times \exp \left[-\left(\alpha_{i}+\beta_{j}\right)\left(x-P_{x}\right)^{2}\right] .
\end{aligned}
$$

Taking into account the binomial theorem, we can actually write

$$
\begin{aligned}
\left(x-A_{x}\right)^{a_{x}}\left(x-B_{x}\right)^{b_{x}}= & \sum_{i_{x}=0}^{a_{x}} \sum_{j_{x}=0}^{b_{x}}\left(\begin{array}{c}
a_{x} \\
i_{x}
\end{array}\right)\left(\begin{array}{c}
b_{x} \\
j_{x}
\end{array}\right)\left(x-P_{x}\right)^{i_{x}+j_{x}} \\
& \times\left(P_{x}-A_{x}\right)^{a_{x}-i_{x}}\left(P_{x}-B_{x}\right)^{b_{x}-j_{x}} .
\end{aligned}
$$

Therefore, $I_{x}\left(a_{x} \mid b_{x}\right)$ can also be expressed as

$$
\begin{aligned}
I_{x}\left(a_{x} \mid b_{x}\right)= & \sum_{i_{x}=0}^{a_{x}} \sum_{j_{x}=0}^{b_{x}}\left(\begin{array}{c}
a_{x} \\
i_{x}
\end{array}\right)\left(\begin{array}{c}
b_{x} \\
j_{x}
\end{array}\right)\left(P_{x}-A_{x}\right)^{a_{x}-i_{x}}\left(P_{x}-B_{x}\right)^{b_{x}-j_{x}} \\
& \times \int \mathrm{d} x \exp \left(i k_{x} x\right) \exp \left[-\left(\alpha_{i}+\beta_{j}\right)\left(x-P_{x}\right)^{2}\right] \\
& \times\left(x-P_{x}\right)^{i_{x}+j_{x}}
\end{aligned}
$$

We also know that the Fourier transform switches from Cartesian to Hermite Gaussians (Č́arsky et al., 1996):

$$
\begin{aligned}
& \int \mathrm{d} x \exp \left(i k_{x} x\right) \exp \left[-\alpha\left(x-A_{x}\right)^{2}\right]\left(x-A_{x}\right)^{l} \\
& =\left(\frac{\pi}{\alpha}\right)^{1 / 2}\left[\frac{i}{2\left(\alpha^{1 / 2}\right)}\right]^{l} H_{l}\left[\frac{k_{x}}{2\left(\alpha^{1 / 2}\right)}\right] \exp \left(-\frac{k_{x}^{2}}{4 \alpha}\right) \exp \left(i k_{x} A_{x}\right),
\end{aligned}
$$

where $H_{l}$ is a Hermite polynomial, namely

$$
H_{l}(x)=(-1)^{l} \exp \left(x^{2}\right) \frac{\mathrm{d}^{l}}{\mathrm{~d} x^{l}} \exp \left(-x^{2}\right) .
$$

Therefore, using relations (23) and (24), equation (22) can be rewritten as

$$
\begin{aligned}
I_{x}\left(a_{x} \mid b_{x}\right)= & \sum_{i_{x}=0}^{a_{x}} \sum_{j_{x}=0}^{b_{x}} \Upsilon_{i_{x}+j_{x}}\left(\begin{array}{c}
a_{x} \\
i_{x}
\end{array}\right)\left(\begin{array}{c}
b_{x} \\
j_{x}
\end{array}\right) \\
& \left(P_{x}-A_{x}\right)^{a_{x}-i_{x}}\left(P_{x}-B_{x}\right)^{b_{x}-j_{x}} \exp \left(i k_{x} P_{x}\right),
\end{aligned}
$$

where $\Upsilon_{i_{x}+j_{x}}$ is defined as follows:

$$
\begin{aligned}
\Upsilon_{i_{x}+j_{x}}= & \left(\frac{\pi}{\alpha_{i}+\beta_{j}}\right)^{1 / 2}\left[\frac{i}{2\left(\alpha_{i}+\beta_{j}\right)^{1 / 2}}\right]^{i_{x}+j_{x}} \\
& \times H_{i_{x}+j_{x}}\left[\frac{k_{x}}{2\left(\alpha_{i}+\beta_{j}\right)^{1 / 2}}\right] \exp \left[-\frac{k_{x}^{2}}{4\left(\alpha_{i}+\beta_{j}\right)}\right] .
\end{aligned}
$$

If we consider the derivative of equation (20) with respect to the nuclear coordinate $A_{x}$, we obtain

$$
\begin{aligned}
& \frac{\partial I_{x}\left(a_{x} \mid b_{x}\right)}{\partial A_{x}}=-a_{x} \int \mathrm{d} x \exp \left(i k_{x} x\right)\left(x-A_{x}\right)^{a_{x}-1}\left(x-B_{x}\right)^{b_{x}} \\
& \quad \times \exp \left[-\left(\alpha_{i}+\beta_{j}\right)\left(x-P_{x}\right)^{2}\right] \\
& \quad+2 \alpha_{i} \int \mathrm{d} x \exp \left(i k_{x} x\right)\left(x-A_{x}\right)^{a_{x}+1}\left(x-B_{x}\right)^{b_{x}} \\
& \quad \times \exp \left[-\left(\alpha_{i}+\beta_{j}\right)\left(x-P_{x}\right)^{2}\right] \\
& \quad+2 \alpha_{i}\left(A_{x}-P_{x}\right) \int \mathrm{d} x \exp \left(i k_{x} x\right)\left(x-A_{x}\right)^{a_{x}}\left(x-B_{x}\right)^{b_{x}} \\
& \quad \times \exp \left[-\left(\alpha_{i}+\beta_{j}\right)\left(x-P_{x}\right)^{2}\right]
\end{aligned}
$$

which, in short notation, can be rewritten as

$$
\begin{aligned}
\frac{\partial I_{x}\left(a_{x} \mid b_{x}\right)}{\partial A_{x}}= & -a_{x} I_{x}\left(a_{x}-1 \mid b_{x}\right)+2 \alpha_{i} I_{x}\left(a_{x}+1 \mid b_{x}\right) \\
& +2 \alpha_{i}\left(A_{x}-P_{x}\right) I_{x}\left(a_{x} \mid b_{x}\right) .
\end{aligned}
$$

In an analogous way, the derivative of equation (20) with respect to the nuclear coordinate $B_{x}$ is

$$
\begin{aligned}
\frac{\partial I_{x}\left(a_{x} \mid b_{x}\right)}{\partial B_{x}}= & -b_{x} I_{x}\left(a_{x} \mid b_{x}-1\right)+2 \beta_{j} I_{x}\left(a_{x} \mid b_{x}+1\right) \\
& +2 \beta_{j}\left(B_{x}-P_{x}\right) I_{x}\left(a_{x} \mid b_{x}\right) .
\end{aligned}
$$

Considering equation (25), the derivative with respect to $A_{x}$ (see supporting information for more details) is

$$
\begin{aligned}
\frac{\partial I_{x}\left(a_{x} \mid b_{x}\right)}{\partial A_{x}}= & \left(\frac{\alpha_{i}}{\alpha_{i}+\beta_{j}}-1\right) a_{x} I_{x}\left(a_{x}-1 \mid b_{x}\right) \\
& +\frac{\alpha_{i}}{\alpha_{i}+\beta_{j}} b_{x} I_{x}\left(a_{x} \mid b_{x}-1\right)+i k_{x} \frac{\alpha_{i}}{\alpha_{i}+\beta_{j}} I_{x}\left(a_{x} \mid b_{x}\right) .
\end{aligned}
$$

In an analogous way, for the derivative of equation (25) with respect to $B_{x}$, we have

$$
\begin{aligned}
\frac{\partial I_{x}\left(a_{x} \mid b_{x}\right)}{\partial B_{x}}= & \left(\frac{\beta_{j}}{\alpha_{i}+\beta_{j}}-1\right) b_{x} I_{x}\left(a_{x} \mid b_{x}-1\right) \\
& +\frac{\beta_{j}}{\alpha_{i}+\beta_{j}} a_{x} I_{x}\left(a_{x}-1 \mid b_{x}\right)+i k_{x} \frac{\beta_{j}}{\alpha_{i}+\beta_{j}} I_{x}\left(a_{x} \mid b_{x}\right) .
\end{aligned}
$$

Since the derivatives $\partial I_{x}\left(a_{x} \mid b_{x}\right) / \partial A_{x}$ given by equations (28) and (30) must be identical, we can equate the right-hand sides of the two relations and obtain

$$
\begin{aligned}
I_{x}\left(a_{x}+1 \mid b_{x}\right)= & {\left[P_{x}-A_{x}+\frac{i k_{x}}{2\left(\alpha_{i}+\beta_{j}\right)}\right] I_{x}\left(a_{x} \mid b_{x}\right) } \\
& +\frac{a_{x}}{2\left(\alpha_{i}+\beta_{j}\right)} I_{x}\left(a_{x}-1 \mid b_{x}\right) \\
& +\frac{b_{x}}{2\left(\alpha_{i}+\beta_{j}\right)} I_{x}\left(a_{x} \mid b_{x}-1\right) .
\end{aligned}
$$

This is the vertical recurrence relation (VRR) for the 'bra' part of the Fourier integral. In the same way, equating the right-hand sides of equations (29) and (31) we get 


$$
\begin{aligned}
I_{x}\left(a_{x} \mid b_{x}+1\right)= & {\left[P_{x}-B_{x}+\frac{i k_{x}}{2\left(\alpha_{i}+\beta_{j}\right)}\right] I_{x}\left(a_{x} \mid b_{x}\right) } \\
& +\frac{a_{x}}{2\left(\alpha_{i}+\beta_{j}\right)} I_{x}\left(a_{x}-1 \mid b_{x}\right) \\
& +\frac{b_{x}}{2\left(\alpha_{i}+\beta_{j}\right)} I_{x}\left(a_{x} \mid b_{x}-1\right)
\end{aligned}
$$

which is the VRR for the 'ket' part of the Fourier integral.

Finally, comparing equations (32) and (33), one can also obtain

$$
I_{x}\left(a_{x} \mid b_{x}+1\right)=I_{x}\left(a_{x}+1 \mid b_{x}\right)+\left(A_{x}-B_{x}\right) I_{x}\left(a_{x} \mid b_{x}\right),
$$

which is the horizontal recurrence relation (HRR).

The starting point for the recurrence formulas (32), (33) and (34) is the Fourier integral of the product that involves only $s$ type Cartesian Gaussian-type functions (i.e. Cartesian GTFs with a total angular momentum equal to zero). This integral is easy to compute:

$$
\begin{aligned}
I_{x}(0 \mid 0) & =\int \mathrm{d} x \exp \left(i k_{x} x\right) \exp \left[-\left(\alpha_{i}+\beta_{j}\right)\left(x-P_{x}\right)^{2}\right] \\
& =\left(\frac{\pi}{\alpha_{i}+\beta_{j}}\right)^{1 / 2} \exp \left[-\frac{k_{x}^{2}}{4\left(\alpha_{i}+\beta_{j}\right)}\right] \exp \left(i k_{x} P_{x}\right) .
\end{aligned}
$$

The recurrence relations obtained above can obviously be extended to the factors $I_{y}\left(a_{y} \mid b_{y}\right)$ and $I_{z}\left(a_{z} \mid b_{z}\right)$ in (19), and therefore equations (32), (33) and (34) can, respectively, be generalized to the three-dimensional case as follows:

$$
\begin{aligned}
& I_{i a}^{j b}\left(\mathbf{a}+\mathbf{1}_{n} \mid \mathbf{b}\right)= {\left[P_{n}-A_{n}+\frac{i k_{n}}{2\left(\alpha_{i}+\beta_{j}\right)}\right] I_{i a}^{j b}(\mathbf{a} \mid \mathbf{b}) } \\
&+\frac{a_{n}}{2\left(\alpha_{i}+\beta_{j}\right)} I_{i a}^{j b}\left(\mathbf{a}-\mathbf{1}_{n} \mid \mathbf{b}\right) \\
&+\frac{b_{n}}{2\left(\alpha_{i}+\beta_{j}\right)} I_{i a}^{j b}\left(\mathbf{a} \mid \mathbf{b}-\mathbf{1}_{n}\right), \\
& I_{i a}^{j b}\left(\mathbf{a} \mid \mathbf{b}+\mathbf{1}_{n}\right)= {\left[P_{n}-B_{n}+\frac{i k_{n}}{2\left(\alpha_{i}+\beta_{j}\right)}\right] I_{i a}^{j b}(\mathbf{a} \mid \mathbf{b}) } \\
&+\frac{a_{n}}{2\left(\alpha_{i}+\beta_{j}\right)} I_{i a}^{j b}\left(\mathbf{a}-\mathbf{1}_{n} \mid \mathbf{b}\right) \\
&+\frac{b_{n}}{2\left(\alpha_{i}+\beta_{j}\right)} I_{i a}^{j b}\left(\mathbf{a} \mid \mathbf{b}-\mathbf{1}_{n}\right), \\
& I_{i a}^{j b}\left(\mathbf{a} \mid \mathbf{b}+\mathbf{1}_{n}\right)=I_{i a}^{j b}\left(\mathbf{a}+\mathbf{1}_{n} \mid \mathbf{b}\right)+\left(A_{n}-B_{n}\right) I_{i a}^{j b}(\mathbf{a} \mid \mathbf{b}),
\end{aligned}
$$

where $n=x, y$ or $z$ and $\mathbf{1}_{n}=\left(\delta_{n x}, \delta_{n y}, \delta_{n z}\right)$ with $\delta$ as the Kronecker delta, and where the starting point is the integral

$$
\begin{aligned}
I_{i a}^{j b}(\mathbf{0} \mid \mathbf{0}) & =E_{i a}^{j b} I_{x}(0 \mid 0) I_{y}(0 \mid 0) I_{z}(0 \mid 0) \\
& =E_{i a}^{j b}\left(\frac{\pi}{\alpha_{i}+\beta_{j}}\right)^{3 / 2} \exp \left[-\frac{k^{2}}{4\left(\alpha_{i}+\beta_{j}\right)}\right] \exp (i \mathbf{k} \cdot \mathbf{P}) .
\end{aligned}
$$

Equation (38) depends only on the nuclear centres of the primitives. Therefore, it can be directly used to evaluate integrals $I$ between contracted basis functions [see equation (10)],

$$
I\left[\mathbf{a} \mid \mathbf{b}+\mathbf{1}_{n}\right]=I\left[\mathbf{a}+\mathbf{1}_{n} \mid \mathbf{b}\right]+\left(A_{n}-B_{n}\right) I[\mathbf{a} \mid \mathbf{b}],
$$

where the square brackets indicate integrals involving contracted basis functions. This relation provides a significant reduction in the computational cost associated with the evaluation of Fourier integrals involving highly contracted Cartesian GTFs.

Finally, since spherical Gaussian-type functions can be written as linear combinations of Cartesian Gaussian-type functions (Schlegel \& Frisch, 1995), it is worth noting that the Obara-Saika recurrence relations can also be trivially used to evaluate Fourier integrals of spherical Gaussian products. In fact, it is enough to use the derived recurrence relations to compute the necessary Fourier integrals of Cartesian Gaussian products, which are afterwards properly combined to obtain the desired integrals involving spherical Gaussians.

\section{Implementation of the Obara-Saika relations for the calculation of structure factors}

\subsection{Structure of the algorithm}

In this section we will briefly illustrate how the OS recurrence relations have been implemented for the computation of structure factors, for example in the program associated with the X-ray constrained ELMO method (Genoni, 2013a,b). The formal structure of the algorithm is presented in Fig. 1, from which it is immediately evident that the implementation requires four nested loops. The outermost loop is over the reflections for which we want to compute the structure factors. The second loop is over the shell pairs, where the term 'shell' is that used in quantum chemistry to indicate a group of contracted Cartesian Gaussian-type functions having the same total angular momentum. It must not be confused with the term normally used in atomic physics to indicate electron shells (i.e. a group of electrons with the same principal quantum number). For this reason we can immediately compute the total angular momentum $m=L_{\alpha}+L_{\beta}$ of the shell pair and, consequently, of the integral. Furthermore, since all the basis functions of a shell are centred on the same nucleus, we can also calculate and store the distance between the nuclei associated with the shell pair under consideration. The third loop is over the equivalent positions in the unit cell to compute the different contributions to the matrix element $I_{\alpha \beta}^{\mathbf{H}}$ [see equation (9)]. In this case, before starting the innermost loop, it is advantageous to compute the exponential and thermal pre-factors $\left[\right.$ i.e. $\exp \left(i 2 \pi \mathbf{t}_{j} \cdot \mathbf{H}\right)$ and $F[P(\mathbf{A}, \mathbf{B})]$ in equation (9), respectively] and the vector $\mathbf{k}$ [see equation (10)]. Finally, the innermost loop runs over all possible pairs of primitives for the considered pair of shells, and the OS recurrence relations really come into play to compute the required integrals. 
For a given pair of primitives, the first step is the computation of the vector $\mathbf{P}$ [see equation (17)] and the starting integral $I_{i a}^{j b}(\mathbf{0} \mid \mathbf{0})$ through equation (39). Note that, for the sake of simplicity and clarity, in Fig. 1 integrals $I_{i a}^{j b}(\mathbf{a} \mid \mathbf{b})$ are indicated as integrals $I(\mathbf{a} \mid \mathbf{b})$. If the total angular momentum $m$ of the shell pair is different from 0 , the vertical recurrence relation expressed by equation (36) is applied to obtain integrals $I_{i a}^{j b}(\mathbf{w} \mid \mathbf{0})$, where $\mathbf{w}=\left(w_{x}, w_{y}, w_{z}\right)$ and $0 \leq w_{x}+w_{y}+w_{z} \leq$ $m$. Afterwards, some of these integrals (i.e. those with $\left.L_{\alpha} \leq w_{x}+w_{y}+w_{z} \leq m\right)$ are contracted incrementally. Obviously, if the total angular momentum $m$ is equal to zero, the integral is immediately contracted.

After completing the innermost loop, all the necessary contracted integrals $I_{i a}^{j b}[\mathbf{w} \mid \mathbf{0}]$ are available and used to complete the loop over the equivalent positions in the unit cell, where the horizontal recurrence relation (40) is applied to compute the target integrals [if the angular momentum of the 'ket' part $\left(L_{\beta}\right)$ is different from zero]. The target integrals are then multiplied by the normalization constants and by the exponential and thermal pre-factors. Finally, the computed integrals are used for the calculation of the desired structure factor [through equation (8)] in the last step of the loop over the reflections. Analysing the structure of the algorithm, it is also worth noting that the procedure is easily parallelizable, which would further increase the computational advantages of the OS approach in quantum crystallography.
Table 1

Mean absolute deviations between structure factors computed in double precision through the OS and MD approaches.

\begin{tabular}{lll}
\hline & cc-pVDZ & cc-pVTZ \\
\hline$\alpha$-Glycine & $1.5 \times 10^{-7}$ & $1.5 \times 10^{-7}$ \\
$L$-Alanine & $2.6 \times 10^{-8}$ & $2.3 \times 10^{-8}$ \\
Salicylic acid & $2.2 \times 10^{-7}$ & $2.3 \times 10^{-7}$ \\
\hline
\end{tabular}

\subsection{Benchmark calculations}

In order to demonstrate the accuracy and robustness of the OS method in the calculation of structure factors, in this subsection we show the results obtained using our in-house Fortran90 program that implements the algorithm described above. To accomplish this task we computed structure factors associated with static electron densities (namely, by setting the anisotropic displacement parameters to 0) calculated at the Hartree-Fock level on the crystal geometries of $\alpha$-glycine (Destro et al., 2000), L-alanine (Destro et al., 1988) and salicylic acid (Munshi \& Guru Row, 2006). Two different basis sets were used, cc-pVDZ and cc-pVTZ (Dunning, 1989). We computed the structure factors corresponding to the reflections deposited with the crystal structures (Destro et al., 2000, 1988; Munshi \& Guru Row, 2006). The values obtained using the OS method were compared with those resulting from the use of the MD approach in Tonto (Jayatilaka \& Grimwood, 2003). The mean absolute discrepancies are shown in Table 1 ,

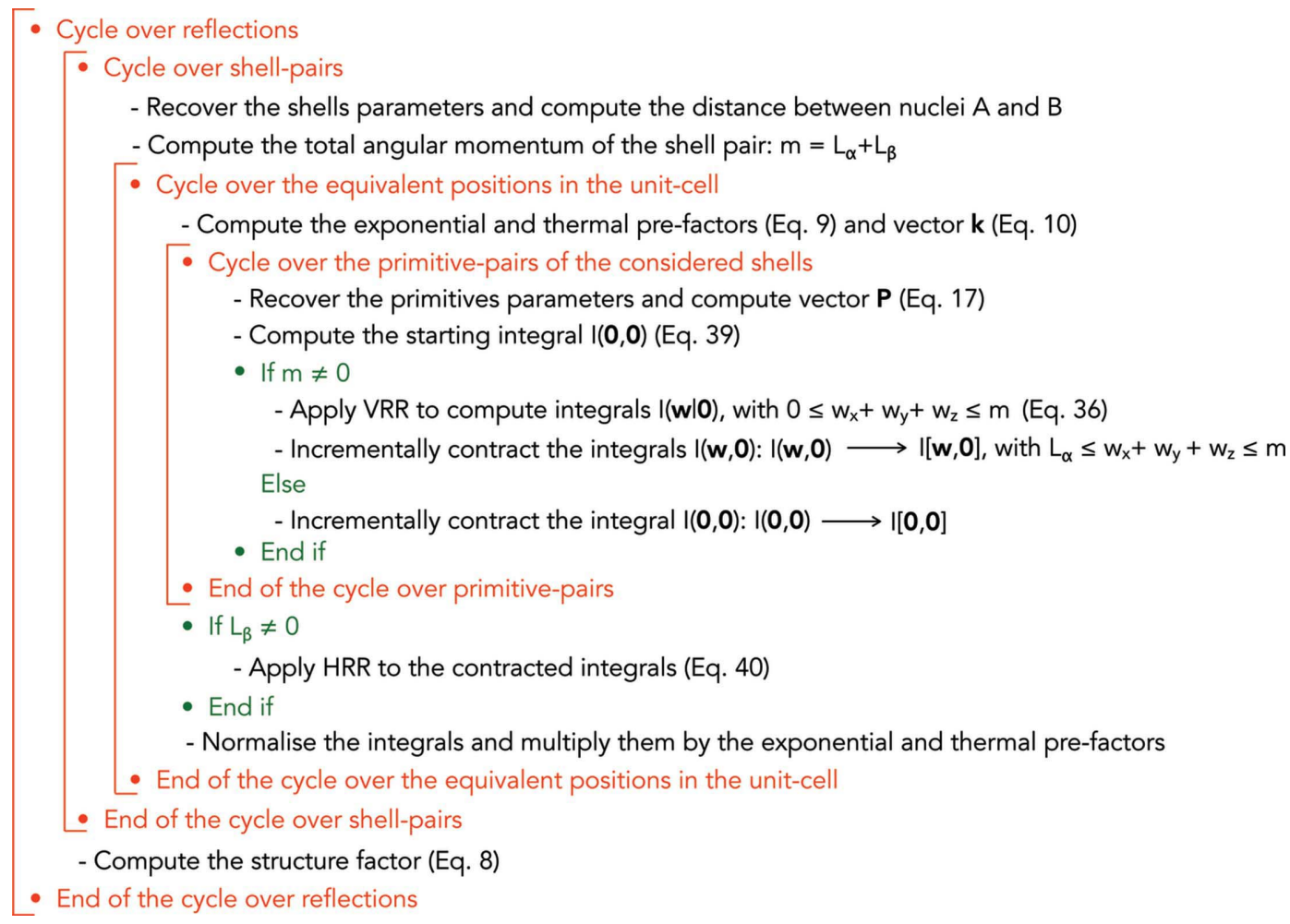

Figure 1

A schematic representation of the algorithm that implements the computation of structure factors through the use of the OS recurrence relations for Fourier integrals of Cartesian Gaussian products. The four nested loops of the algorithm are highlighted in red and by vertical lines at the side, while the 'if' constructs are highlighted in green. For the sake of simplicity and clarity, in this figure, the primitive integrals $I(\mathbf{a} \mid \mathbf{b})$ correspond to the primitive integrals $I_{i a}^{j b}(\mathbf{a} \mid \mathbf{b})$ in the text. 
Table 2

Timings (in seconds) associated with the calculation of the structure factors by means of the OS recurrence relations: global CPU time for the complete set of reflections, and average time for the computation of a single structure factor.

The recorded timings were obtained running our in-house Fortran 90 code (statically compiled with version 18.0.3 of the Intel Fortran Compiler) on an Intel Xeon Gold $61302.1 \mathrm{GHz}$ processor. NBF is the number of basis functions. The numbers in parentheses in the leftmost column are the numbers of considered reflections.

\begin{tabular}{|c|c|c|c|c|c|c|}
\hline & \multicolumn{3}{|c|}{ cc-pVDZ } & \multicolumn{3}{|c|}{ cc-pVTZ } \\
\hline & NBF & $\begin{array}{l}\text { Global } \\
\text { time (s) }\end{array}$ & $\begin{array}{l}\text { Average } \\
\text { time (s) }\end{array}$ & NBF & $\begin{array}{l}\text { Global } \\
\text { time (s) }\end{array}$ & $\begin{array}{l}\text { Average } \\
\text { time (s) }\end{array}$ \\
\hline$-\mathrm{Gl}$ & 100 & 62.808 & 0.016 & 25 & 298.316 & 0.078 \\
\hline L-Alanine (2519) & 125 & 62.375 & 0.025 & 315 & 296.123 & 0.118 \\
\hline Salicylic acid (5704) & 180 & 348.947 & 0.061 & 440 & 1238.053 & 0.217 \\
\hline
\end{tabular}

while the values of the single structure factors obtained through the OS and MD approaches are provided in separate files in the supporting information. The two methods and the associated programs provide practically the same results.

For the sake of completeness, in Table 2 we also report the timings associated with the calculation of structure factors using the OS approach. The computation of single structure factors is already quite fast, but it is also worth noting that our current in-house program has not been optimized and, above all, has not been parallelized yet. Therefore, the speed of structure-factor calculations by means of the OS recurrence relations can be increased further.

\section{Conclusions}

We have shown that the Obara-Saika approach can easily be used for efficient analytical computation of structure factors in quantum crystallography. Unlike the pioneering techniques (McWeeny, 1953; Chandler \& Spackman, 1978; Barua \& Weyrich, 1986) based on case-by-case tabular enumerations of analytical results, the strategy described in this work makes use of recurrence relations and, for this reason, is conceptually close to the method relying on the extension of the McMurchie-Davidson recursion relationships introduced by Jayatilaka (1994). The difference between the OS and MD approaches is that the starting point of the latter consists of writing the products of Cartesian Gaussian-type functions as a linear combination of one-centre Hermite Gaussians, which enables the reduction of the integrations over two-centre functions to integrations over a set of one-centre functions. Recurrence relations are obviously advantageous from the computational point of view and nowadays are routinely used in modern quantum chemistry programs, although it is also worth bearing in mind that they may be subject to 'digital erosion' issues, as investigated by Barnett (2002) and, more recently, by Bailey \& Borwein (2015).

Finally, it is important to note that, in this article, we have only shown how the OS method can be applied to the calculation of X-ray structure factors. However, the presented strategy can also be easily extended to the calculation of magnetic structure factors associated with polarized neutron diffraction data and to the computation of Compton profiles. In other words, the applicability of the OS approach is not limited only to HAR or XCW methods, and in the future it could indeed be exploited in all those techniques of quantum crystallography that start from a wavefunction or density matrix ansatz. For example, it could be used in the context of the refinement strategies recently proposed by Gillet and coworkers (Guedidda, Yan \& Gillet, 2018; Guedidda, Yan, Kibalin et al., 2018; De Bruyne \& Gillet, 2020) to obtain charge- or spin-resolved density matrices and densities, which could subsequently be analysed by means of the novel sophisticated approaches of quantum chemical topology (Gatti et al., 2015, 2017; Macetti et al., 2018).

\section{Funding information}

The following funding is acknowledged: the French Research Agency (ANR) for financial support of the Young Researcher Project QuMacroRef (grant No. ANR-17-CE29-0005-01 to Alessandro Genoni).

\section{References}

Bailey, D. H. \& Borwein, J. M. (2015). Mathematics 3, 337-367.

Barnett, M. P. (2002). Theor. Chem. Acc. 107, 241-245.

Barua, M. \& Weyrich, W. (1986). Acta Cryst. A42, 257-261.

Boys, S. F. (1950). Proc. R. Soc. London A, 200, 542-554.

Bytheway, I., Grimwood, D. J., Figgis, B. N., Chandler, G. S. \& Jayatilaka, D. (2002). Acta Cryst. A58, 244-251.

Bytheway, I., Grimwood, D. J. \& Jayatilaka, D. (2002). Acta Cryst. A58, 232-243.

Capelli, S. C., Bürgi, H.-B., Dittrich, B., Grabowsky, S. \& Jayatilaka, D. (2014). IUCrJ, 1, 361-379.

Čársky, P., Hrouda, V. \& Polášek, M. (1996). Theor. Chim. Acta, 93, 49-59.

Casati, N., Genoni, A., Meyer, B., Krawczuk, A. \& Macchi, P. (2017). Acta Cryst. B73, 584-597.

Chandler, G. S. \& Spackman, M. A. (1978). Acta Cryst. A34, 341-343.

Clementi, E. \& Corongiu, G. (1989). Modern Techniques in Computational Chemistry: MOTECC-89, edited by E. Clementi, pp. 243-306. Leiden: ESCOM.

Clinton, W. L., Frishberg, C. A., Massa, L. J. \& Oldfield, P. L. (1973). Int. J. Quantum Chem. 7, 505-514.

Clinton, W. L. \& Massa, L. J. (1972). Phys. Rev. Lett. 29, 1363-1366. Coppens, P. (1997). X-ray Charge Densities and Chemical Bonding. Oxford University Press.

Coppens, P., Willoughby, T. V. \& Csonka, L. N. (1971). Acta Cryst. A27, 248-256.

De Bruyne, B. \& Gillet, J.-M. (2020). Acta Cryst. A76, 1-6.

Destro, R., Marsh, R. E. \& Bianchi, R. (1988). J. Phys. Chem. 92, 966973.

Destro, R., Roversi, P., Barzaghi, M. \& Marsh, R. E. (2000). J. Phys. Chem. A, 104, 1047-1054.

Dos Santos, L. H. R., Genoni, A. \& Macchi, P. (2014). Acta Cryst. A70, 532-551.

Dunning, T. H. (1989). J. Chem. Phys. 90, 1007-1023.

Fugel, M., Jayatilaka, D., Hupf, E., Overgaard, J., Hathwar, V. R., Macchi, P., Turner, M. J., Howard, J. A. K., Dolomanov, O. V., Puschmann, H., Iversen, B. B., Bürgi, H.-B. \& Grabowsky, S. (2018). IUCrJ, 5, 32-44.

Fugel, M., Ponomarenko, M. V., Hesse, M. F., Malaspina, L. A., Kleemiss, F., Sugimoto, K., Genoni, A., Röschenthaler, G.-V. \& Grabowsky, S. (2019). Dalton Trans. 48, 16330-16339.

Gao, C., Genoni, A., Gao, S., Jang, S., Soncini, A. \& Overgaard, J. (2019). Nat. Chem. doi: 10.1038/s41557-019-0387-6. 
Gatti, C., Macetti, G. \& Lo Presti, L. (2017). Acta Cryst. B73, 565583.

Gatti, C., Orlando, A. M. \& Lo Presti, L. (2015). Chem. Sci. 6, 38453852.

Genoni, A. (2013a). J. Phys. Chem. Lett. 4, 1093-1099.

Genoni, A. (2013b). J. Chem. Theory Comput. 9, 3004-3019.

Genoni, A. (2017). Acta Cryst. A73, 312-316.

Genoni, A., Bučinský, L., Claiser, N., Contreras-García, J., Dittrich, B., Dominiak, P. M., Espinosa, E., Gatti, C., Giannozzi, P., Gillet, J.-M., Jayatilaka, D., Macchi, P., Madsen, A. Ø., Massa, L. J., Matta, C. F., Merz, K. M. Jr, Nakashima, P. N. H., Ott, H., Ryde, U., Schwarz, K., Sierka, M. \& Grabowsky, S. (2018). Chem. Eur. J. 24, 10881-10905.

Genoni, A., Dos Santos, L. H. R., Meyer, B. \& Macchi, P. (2017). IUCrJ, 4, 136-146.

Genoni, A., Franchini, D., Pieraccini, S. \& Sironi, M. (2018). Chem. Eur. J. 24, 15507-15511.

Genoni, A., Macetti, G., Franchini, D., Pieraccini, S. \& Sironi, M. (2019). Acta Cryst. A75, 778-797.

Genoni, A. \& Meyer, B. (2016). Adv. Quantum Chem. 73, 333-362.

Giacovazzo, C. (2002). Fundamentals of Crystallography, 2nd ed. Oxford University Press.

Gill, P. M. W. (1994). Adv. Quantum Chem. 25, 141-205.

Gillet, J.-M. (2007). Acta Cryst. A63, 234-238.

Gillet, J.-M., Becker, P. J. \& Cortona, P. (2001). Phys. Rev. B, 63, 235115.

Grabowsky, S., Genoni, A. \& Bürgi, H.-B. (2017). Chem. Sci. 8, 41594176.

Grimwood, D. J., Bytheway, I. \& Jayatilaka, D. (2003). J. Comput. Chem. 24, 470-483.

Grimwood, D. J. \& Jayatilaka, D. (2001). Acta Cryst. A57, 87-100.

Gueddida, S., Yan, Z. \& Gillet, J.-M. (2018). Acta Cryst. A74, 131-142.

Gueddida, S., Yan, Z., Kibalin, I., Voufack, A. B., Claiser, N., Souhassou, M., Lecomte, C., Gillon, B. \& Gillet, J.-M. (2018). J. Chem. Phys. 148, 164106.

Hansen, N. K. \& Coppens, P. (1978). Acta Cryst. A34, 909-921.

Helgaker, T., Jørgensen, P. \& Olsen, J. (2000). Molecular Electronic Structure Theory. Chichester: John Wiley and Sons Ltd.

Hirshfeld, F. L. (1971). Acta Cryst. B27, 769-781.

Hudák, M., Jayatilaka, D., Perašínová, L., Biskupič, S., Kožíšek, J. \& Bučinský, L. (2010). Acta Cryst. A66, 78-92.

Jayatilaka, D. (1994). Chem. Phys. Lett. 230, 228-230.

Jayatilaka, D. (1998). Phys. Rev. Lett. 80, 798-801.

Jayatilaka, D. (2012). Modern Charge-Density Analysis, edited by C. Gatti \& P. Macchi, pp. 213-257. Dordrecht: Springer Netherlands.

Jayatilaka, D. \& Dittrich, B. (2008). Acta Cryst. A64, 383-393.

Jayatilaka, D. \& Grimwood, D. J. (2001). Acta Cryst. A57, 76-86.

Jayatilaka, D. \& Grimwood, D. J. (2003). Computational Science ICCS 2003, edited by P. M. A. Sloot, D. Abramson, A. V. Bogdanov, J. J. Dongarra, A. Y. Zomaya \& Y. E. Gorbachev, pp. 142-151. Berlin, Heidelberg: Springer-Verlag.

Kurki-Suonio, K. (1977). Isr. J. Chem. 16, 132-136.
Macetti, G., Lo Presti, L. \& Gatti, C. (2018). J. Comput. Chem. 39, 587-603.

Malaspina, L. A., Wieduwilt, E. K., Bergmann, J., Kleemiss, F., Meyer, B., Ruiz-López, M. F., Pal, R., Hupf, E., Beckmann, J., Piltz, R. O., Edwards, A. J., Grabowsky, S. \& Genoni, A. (2019). J. Phys. Chem. Lett. 10, 6973-6982.

Massa, L. \& Matta, C. F. (2018). J. Comput. Chem. 39, 1021-1028.

McMurchie, L. E. \& Davidson, E. R. (1978). J. Comput. Phys. 26, 218-231.

McWeeny, R. (1953). Acta Cryst. 6, 631-637.

Meyer, B. \& Genoni, A. (2018). J. Phys. Chem. A, 122, 8965-8981.

Meyer, B., Guillot, B., Ruiz-Lopez, M. F. \& Genoni, A. (2016a). J. Chem. Theory Comput. 12, 1052-1067.

Meyer, B., Guillot, B., Ruiz-Lopez, M. F., Jelsch, C. \& Genoni, A. (2016b). J. Chem. Theory Comput. 12, 1068-1081.

Michael, J. R. \& Koritsanszky, T. (2015). J. Math. Chem. 53, 250-259.

Moreno Carrascosa, A., Yong, H., Crittenden, D. L., Weber, P. \& Kirrander, A. (2019). J. Chem. Theory Comput. 15, 2836-2846.

Munshi, P. \& Guru Row, T. N. (2006). Acta Cryst. B62, 612-626.

Northey, T. \& Kirrander, A. (2019). J. Phys. Chem. A, 123, 3395-3406.

Northey, Y., Zotev, N. \& Kirrander, A. (2014). J. Chem. Theory Comput. 10, 4911-4920.

Novara, R. F., Genoni, A. \& Grabowsky, S. (2018). ChemViews, doi: 10.1002/chemv.201800066.

Obara, S. \& Saika, A. (1986). J. Chem. Phys. 84, 3963-3974.

Obara, S. \& Saika, A. (1988). J. Chem. Phys. 89, 1540-1559.

Reine, S., Helgaker, T. \& Lindh, R. (2012). WIREs Comput. Mol. Sci. 2, 290-303.

Schlegel, H. B. \& Frisch, M. J. (1995). Int. J. Quantum Chem. 54, 8387.

Schmider, H., Smith, V. H. Jr \& Weyrich, W. (1992). J. Chem. Phys. 96, 8986-8994.

Schwarzenbach, D. (1996). Crystallography. Chichester: John Wiley and Sons Ltd.

Stewart, R. F. (1969). J. Chem. Phys. 51, 4569-4577.

Stewart, R. F. (1976). Acta Cryst. A32, 565-574.

Stoll, H., Wagenblast, G. \& Preuß, H. (1980). Theor. Chim. Acta, 57, 169-178.

Tanaka, K. (1988). Acta Cryst. A44, 1002-1008.

Thomsen, M. K., Nyvang, A., Walsh, J. P. S., Bunting, P. C., Long, J. R., Neese, F., Atanasov, M., Genoni, A. \& Overgaard, J. (2019). Inorg. Chem. 58, 3211-3218.

Tsirelson, V. (2018). J. Comput. Chem. 39, 1029-1037.

Tsirelson, V. G. \& Ozerov, R. P. (1996). Electron Density and Bonding in Crystals: Principles, Theory and X-ray Diffraction Experiments in Solid State Physics and Chemistry. Boca Raton: CRC Press.

Wall, M. E. (2016). IUCrJ, 3, 237-246.

Woińska, M., Grabowsky, S., Dominiak, P. M., Woźniak, K. \& Jayatilaka, D. (2016). Sci. Adv. 2, e1600192.

Woińska, M., Jayatilaka, D., Spackman, M. A., Edwards, A. J., Dominiak, P. M., Woźniak, K., Nishibori, E., Sugimoto, K. \& Grabowsky, S. (2014). Acta Cryst. A70, 483-498. 